\title{
Ten-Year Follow-Up after Bilateral Submacular Neovascular Membrane Removal in a Case of Autosomal Recessive Bestrophinopathy
}

\author{
Carlos A. Moreira Jr. ${ }^{a}$ b Carlos A. Moreira-Neto ${ }^{b}$ \\ Mario Junqueira Nobrega ${ }^{c}$ Eduardo Cunha de Souza ${ }^{d}$

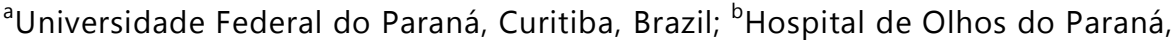

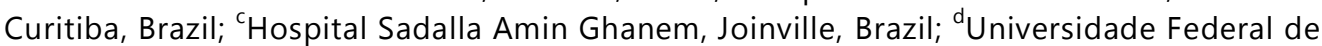 \\ São Paulo, São Paulo, Brazil
}

\section{Keywords}

Bestrophinopathy · Best dystrophy · Retina · Vitrectomy

\begin{abstract}
Herein, we report the case of an 8-year-old girl who presented in December 2000 with a submacular neovascular membrane in the right eye, with a clinical diagnosis of Best disease. At that time, she underwent pars plana vitrectomy (PPV) with removal of the subretinal choroidal neovascularization (CNV). Her vision improved from 20/200 to 20/25. Four years later, a new CNV developed in the other eye. Initially, she underwent unsuccessful photodynamic therapy. As her vision worsened, she underwent a second, this time successful, PPV with membrane removal in the left eye, with vision improving to 20/30. Ten years later, she returned complaining of vision loss over the last year. Her vision was 20/200 OU, and optical coherence tomography demonstrated very large intraretinal cystoid spaces resembling bilateral macular schisis. Four ranibizumab injections as well as dorzolamide eye drops were tried, both without success. Finally, she underwent PPV with internal limiting membrane peeling and gas-fluid exchange in the left eye. One month later, the macula appeared flat and vision had improved to 20/60. The same procedure was performed 1 year later for the right eye, with vision improving to 20/80. One year later, mild cystic spaces developed again in both eyes, although much smaller than previously observed. Her vision remained stable.
\end{abstract}




\section{Case Reports in Ophthalmology}

\section{Introduction}

Best dystrophy is known to be related to an autosomal dominant inheritance caused by mutation in the BEST1 gene. However, there is another entity called autosomal recessive bestrophinopathy (ARB), described by Burgess et al. [1] in 2008 as an autosomal recessive condition associated with biallelic mutations in the BEST1 gene, with either homozygous or compound heterozygous sequence variants. In both Best dystrophy and ARB, yellow epithelial retinal and deep retinal pigment deposits are present, and there is reduced light-rise on electrooculogram. ARB can also be associated with choroidal neovascularization (CNV) early in its course [2].

This report describes a challenging case with long-term follow-up of bestrophinopathy and innovative, multimodal management.

\section{Case Report}

In December 2000, an 8-year-old Caucasian girl presented with a sudden loss of vision in her right eye. Visual acuity was 20/200 in the right eye and 20/20 in the left. Fundus examination revealed a pale, dot-like, subretinal lesion inferior to the foveola with subretinal fluid and subretinal hemorrhage along the inferior arcade (Fig. 1a). In the left eye, a yellowish subretinal material was present (Fig. 1c). Fluorescein angiography showed an area of hyperfluorescence with leakage at the center of the macula of the right eye, indicating the presence of a subretinal neovascular membrane (Fig. 1b). The left eye showed an area of hyperfluorescence without leakage, indicating staining of the retinal pigment epithelium (RPE) both around and inferior to the foveal center (Fig. 1d). A Stratus optical coherence tomography (OCT) (Carl Zeiss, Germany) performed at that time demonstrated intra- and subretinal fluid in the macula of the right eye.

Electrophysiological tests were performed, demonstrating a full-field electroretinogram with normal cone pathway responses but reduced rod responses. Electrooculogram was clearly abnormal, with an Arden ratio of 1:1 in the right eye and 1:2 in the left (online suppl. figure, see www.karger.com/doi/10.1159/000473696). At that time, Best dystrophy was the differential diagnosis. The relevant family history was negative and family screening was unremarkable.

In the absence of current-day options, i.e., photodynamic therapy (PDT) and anti-vascular endothelial growth factor (VEGF) agents, in December 2000, the therapeutic procedure of choice for such a case (type 2 subretinal membrane) was the removal of the submacular membrane through a 20-G pars plana vitrectomy (PPV). A successful removal was performed, and 3 months after surgery, she had 20/25 vision in the right eye. Postoperative fluorescein angiography revealed an area of staining fluorescence around the fovea, with no leakage.

In July 2004 she returned showing an excellent result in the right eye, maintaining her postoperative 20/25 vision (Fig. 2a, b). On the other hand, the left eye had undergone a sudden decrease in vision, with a clinical picture resembling that of the right eye noticed 4 years earlier. Fluorescein angiography revealed the presence of a submacular neovascular membrane with hyperfluorescence and leakage at the foveal center (Fig. 2d), and Stratus OCT examination clearly revealed a type 2 membrane (Fig. 2e). At that time, PDT with verteporfin was performed without success. One month later, the subretinal membrane had grown, showing more subretinal hemorrhage. As her vision continued to deteriorate, she under- 
went a PPV with subretinal membrane removal in the left eye. Three months later, her vison was $20 / 30$ in the left eye, showing no signs of subretinal membrane.

Ten years later she returned, complaining of central vision deterioration in both eyes over the previous 12 months. Vision was 20/200 in both eyes. Fundus color retinography (Fig. 3a, b) and autofluorescence retinography were performed on both eyes, showing an area of hypoautofluorescence covering most of the posterior pole in both eyes. Spectral domain OCT revealed large amounts of fluid with many cystic spaces and appearance of retinoschisis in both eyes (Fig. 3c, e). A multifocal electroretinogram was performed showing decreased $b$ waves in both eyes, being worse in the right eye.

In the beginning of 2014, she had tried 4 bevacizumab injections in each eye and dorzolamide eye drops for the last 6 months, both without success.

After discussion with the patient (then aged 22) and her parents, a decision was made on an experimental, multimodal approach: another PPV with removal of the internal limiting membrane (ILM) plus $\mathrm{C}_{3} \mathrm{~F}_{8}$ gas injection in the left eye. Four weeks after surgery, all retinal fluid had disappeared and her vision was 20/60. Four weeks later, she began to have intraretinal fluid again (Fig. 3d, f), although at a much lower level. At this point, a genetic examination to test her for bestrophin protein was requested, as ARB was suspected. These tests, performed by the Laboratory of Genetics of the Federal University of São Paulo, Brazil, confirmed a homozygous variation in the BEST1 gene (splice site c.768+1G>A).

The vision in the left eye remained stable and the cystic space did not enlarge, so 1 year later, the patient decided to have the same procedure in the right eye. As with the left eye, all fluid resolved and the cystic spaces disappeared for a few months. Three months after surgery, fluid started to appear again, but at a much lower level than before surgery, and has remained so until the present, 1 year after surgery. The online supplementary figure shows preoperative and 1-year postoperative OCT for the right eye.

\section{Discussion}

The patient described here presented with CNV under the foveal area in one eye at the age of 8 , and 4 years later developed the same condition in the other eye. At that time, the only treatment available was surgical removal of the neovascular membrane through PPV. This procedure was done in both eyes, with favorable results. PDT was applied in the second eye without success. Therefore, the left eye also underwent PPV and membrane removal. Anti-VEGF therapy was not available at that time, and recent studies report good results in this type of patient [3].

ARB onset usually occurs early in life, the disease presenting with decrease visual acuity. Other clinical signs of this condition are diffuse RPE dysfunction, intraretinal and subretinal fluid, and atypical subfoveal vitelliform lesions associated with extramacular and extrafoveal subretinal deposits [1]. A transmembrane protein primarily expressed in the basolateral membrane of the RPE and named bestrophin-1 is encoded in BEST1. The functional role of bestrophin-1 within the RPE remains uncertain, with possible functions as a $\mathrm{Ca}^{2+}$-activated $\mathrm{Cl}-$ channel, a regulator of voltage-gated $\mathrm{Ca}^{2+}$ channels, or as a $\mathrm{HCO}_{3}-$ channel. It is postulated that before the photoreceptor outer segments are shed, large amounts of osmolytes are released which adversely influence the role of bestrophin in RPE cell membranes, leading to faulty phagocytosis and disrupted fluid transport across the RPE [4]. This may explain the accumulation of lipofuscin and the development of cystoid macular edema. The rate of VEGF 
production from RPE cells is also thought to depend on the activity of voltage-dependent L-type $\mathrm{Ca}^{2+}$ channels and may be responsible for the early occurrence of CNV in ARB [5].

Gerth et al. [6] described elongated and thickened photoreceptor outer segments together with thin processes bridging the space between the photoreceptor and RPE layers in an 11-year-old boy with ARB. Iannaccone et al. [2] found that bilateral intravitreal bevacizumab treatment in a 6-year-old child with ARB and associated CNV in one eye did not resolve the fluid fully, although the CNV responded favorably. This is similar to our experience, where the classic CNV was no longer active on fluorescein angiography, but the intraretinal spaces persisted on spectral domain OCT. These separations (schisis) in between many retinal layers connected by columns in both eyes are also OCT features seen in juvenile $\mathrm{X}$-linked retinoschisis. The same features were found in our patient. The fluid in the intraretinal space did not seem to respond to anti-VEGF agents, given that there did not appear to be any benefit in the patient following 4 injections of bevacizumab.

The source of the intraretinal fluid is unknown. Therefore, current OCT-driven treatment protocols applicable to neovascular age-related macular degeneration [7, 8] advocating treatment until complete resolution of fluid is achieved [9] may not be appropriate in $\mathrm{CNV}$ associated with causes other than age-related macular degeneration. Besides anti-VEGF, treatment with PDT has been reported for choroidal neovascular membrane associated with Best vitelliform dystrophy [10]. On the other hand, the good temporary response to PPV and ILM peeling plus gas was encouraging for both the treatment team and the patient. Within 2 weeks, all fluid disappeared and vision improved.

This case highlights the successful outcome of bilateral, consecutive PPV with subretinal neovascularization removal and subsequent ILM peeling with gas endotamponade in a case of bestrophinopathy, and emphasizes the importance of a genetic test as a diagnostic tool in patients with macular dystrophies. This appears to be the longest follow-up reported in the literature to date for a case of ARB.

\section{Statement of Ethics}

All authors declare that have no significant competing financial, professional, or personal interests that might have influenced the performance or presentation of the work described in this paper.

\section{Disclosure Statement}

There are no conflicts of interest to declare from any of the authors.

\section{References}

1 Burgess R, Millar ID, Leroy BP, Urquhart JE, Fearon IM, De Baere E, Brown PD, Robson AG, Wright GA, Kestelyn P, Holder GE, Webster AR, Manson FD, Black GC: Biallelic mutation of BEST1 causes a distinct retinopathy in humans. Am J Hum Genet 2008;82:19-31.

-2 Iannaccone A, Kerr NC, Kinnick TR, Calzada JI, Stone EM: Autosomal recessive Best vitelliform macular dystrophy: report of a family and management of early-onset neovascular complications. Arch Ophthalmol 2011;129:211-217.

-3 Madhusudhan S, Hussain A, Sahni JN: Value of anti-VEGF treatment in choroidal neovascularization associated with autosomal recessive bestrophinopathy. Digit J Ophthalmol 2013;19:59-63.

4 Xiao Q, Hartzell HC, Yu K: Bestrophins and retinopathies. Pflugers Arch 2010;460:559-569. 


\section{Case Reports in Ophthalmology}

\begin{tabular}{l|l}
\hline Case Rep Ophthalmol 2017;8:265-270 \\
\hline DOI: $10.1159 / 000473696$ & $\begin{array}{l}\text { C } 2017 \text { The Author(s). Published by S. Karger AG, Basel } \\
\text { www.karger.com/cop }\end{array}$ \\
\hline
\end{tabular}

Moreira Jr. et al.: Ten-Year Follow-Up after Bilateral Submacular Neovascular Membrane Removal in a Case of Autosomal Recessive Bestrophinopathy

5 Rosenthal R, Heimann H, Agostini H, Martin G, Hansen LL, Strauss O: $\mathrm{Ca}^{2+}$ channels in retinal pigment epithelial cells regulate vascular endothelial growth factor secretion rates in health and disease. Mol Vis 2007;13:443-456.

-6 Gerth C, Zawadzki RJ, Werner JS, Héon E: Detailed analysis of retinal function and morphology in a patient with autosomal recessive bestrophinopathy (ARB). Doc Ophthalmol 2009;118:239-246.

-7 Fung AE, Lalwani GA, Rosenfeld PJ, Dubovy SR, Michels S, Feuer WJ, Puliafito CA, Davis JL, Flynn HW Jr, Esquiabro M: An optical coherence tomography-guided, variable dosing regimen with intravitreal ranibizumab (Lucentis) for neovascular age-related macular degeneration. Am J Ophthalmol 2007;143: 566-583.

8 Davis J, Olsen TW, Stewart M, Sternberg P Jr: How the comparison of age-related macular degeneration treatments trial results will impact clinical care. Am J Ophthalmol 2011;152:509-514.

-9 Rishi E, Rishi P, Mahajan S: Intravitreal bevacizumab for choroidal neovascular membrane associated with Best's vitelliform dystrophy. Indian J Ophthalmol 2010;58:160-162.

10 Rishi P, Sharma T, Gopal L: Photodynamic therapy for childhood choroidal neovascular membrane associated with Best's vitelliform dystrophy. Retin Cases Brief Rep 2009;3:288-292.
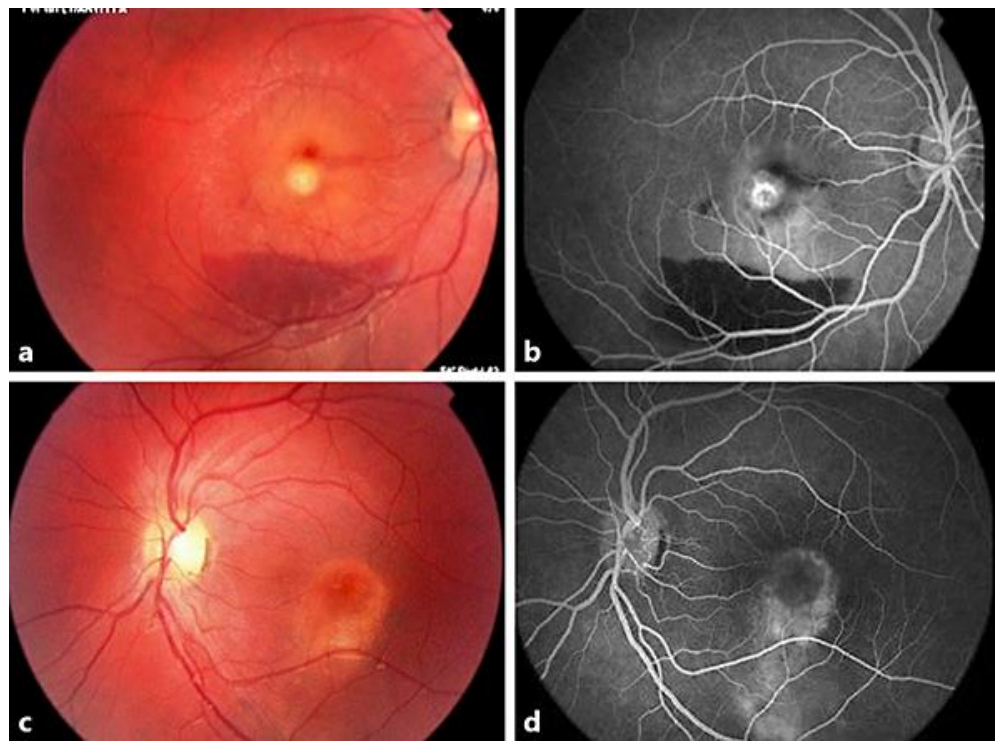

Fig. 1. a Fundus examination revealed a pale, dot-like, subretinal lesion inferior to the foveola with subretinal fluid and subretinal hemorrhage along the inferior arcade. b Fluorescein angiography showed an area of hyperfluorescence with leakage at the center of the macula of the right eye, indicating the presence of a subretinal neovascular membrane. c Fundus examination in the left eye revealed a yellowish subretinal material. $\mathbf{d}$ The left eye showed an area of hyperfluorescence without leakage, indicating staining of the retinal pigment epithelium both around and inferior to the foveal center. 


\section{Case Reports in Ophthalmology} Case Rep Ophthalmol 2017;8:265-270 DOI: $10.1159 / 000473696$

C 2017 The Author(s). Published by S. Karger AG, Basel www.karger.com/cop

Moreira Jr. et al.: Ten-Year Follow-Up after Bilateral Submacular Neovascular Membrane Removal in a Case of Autosomal Recessive Bestrophinopathy

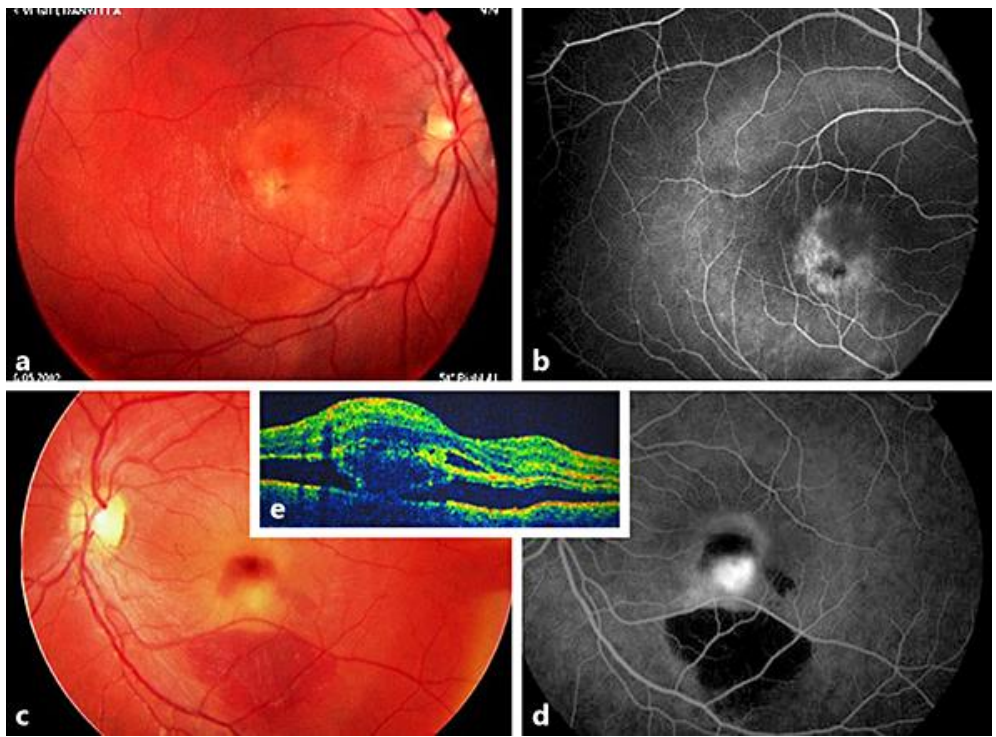

Fig. 2. a, b Fundus examination (a) and fluorescein angiography (b) of the right eye show no more leakage in the right eye. $\mathbf{c}$, $\mathbf{d}$ Fundus examination (c) and fluorescein angiography (d) of the left eye showed a submacular neovascular membrane with hyperfluorescence and leakage at the foveal center. e Stratus optical coherence tomography examination clearly revealed a type 2 membrane.
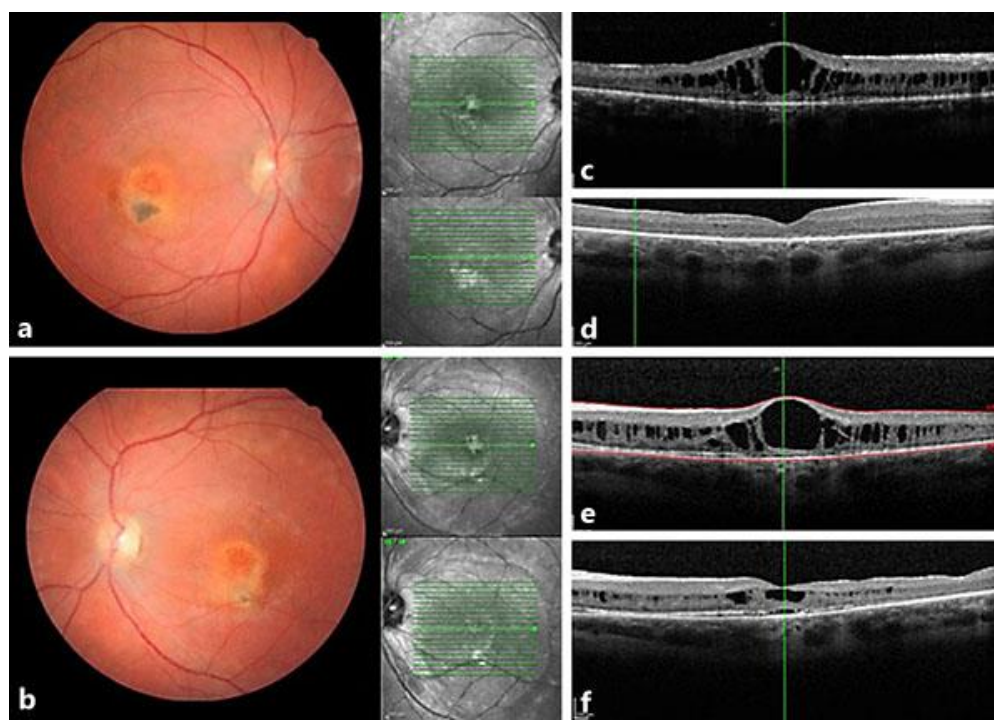

Fig. 3. a, b Fundus color retinographies were done for both eyes, showing a central macular yellow deep lesion in both eyes. c, e Spectral domain optical coherence tomography revealed large amounts of fluid with many cystic spaces and a retinoschisis appearance in both eyes before PPV. $\mathbf{d}$, $\mathbf{f}$ Spectral domain optical coherence tomography b scan showed much less fluid after PPV. 